\title{
Berechnung von Stromlinien im Einzugsbereich von Horizontalfilterbrunnen bei der Uferfiltration
}

\author{
Ernest Mayr · Tobias Gauster - Reinhard Perfler
}

Online publiziert: 15. Juli 2019

(C) Der/die Autor(en) 2019

\begin{abstract}
Zusammenfassung In diesem Artikel ist die Methodik zur Ausweisung von Brunneneinzugsgebieten und zur Abschätzung von Fließzeiten auf Basis eines 3D-stationären Grundwassermodells zusammengestellt. Zusätzlich ist die Methodik zur Erfassung von Quellen (Wasserherkunft) innerhalb eines Brunneneinzugsgebiets zur Abschätzung der Wasserzusammensetzung im Brunnen dargestellt. Folgt man in einem (zeitlich invarianten) Geschwindigkeitsfeld stets den Richtungsvektoren der Wasserbewegung, so entspricht der zurückgelegte Weg einer Stromlinie. Hat man Kenntnis über das dreidimensionale Geschwindigkeitsfeld eines Grundwasserleiters, das jedem beliebigen Punkt innerhalb des stationären Feldes einen Geschwindigkeitsvektor zuordnet, kann für jeden dieser Punkte genau eine einzige Stromlinie bestimmt werden, die durch diesen Punkt geht. Fasst man alle Stromlinien zusammen, die $\mathrm{zu}$ einer Senke (z. B. Brunnen) gehen, so gelangt man zu einer räumlichen Abgrenzung des Einzugsgebiets dieser Senke. Erfasst man alle Quellen eines Einzugsgebiets, kann daraus die Wasserzusammensetzung im Brunnen (Senke) abgeleitet werden.
\end{abstract}

\section{Schlüsselwörter}

Brunneneinzugsgebiet · Uferfiltration · Aufenthaltszeit

\section{Calculation of streamlines in the catchment area of horizontal filter wells for bank filtration}

Abstract This article describes the

DI E. Mayr $(\bowtie) \cdot$ DI T. Gauster ·

PD DI Dr. R. Perfler

Department

Wasser-Atmosphäre-Umwelt,

Institut für Siedlungswasserbau, Industriewasserwirtschaft und

Gewässerschutz, Universität

für Bodenkultur Wien,

Muthgasse 18, 1190 Wien, Österreich

ernest.mayr@boku.ac.at methodology for designating well catchmerstationären Zustände ermöglichen jeareas and estimating flow times based on a 3D stationary groundwater model. In addition, a methodology is presented for capturing sources (origin of water) within a well catchment area for estimating the water composition in the respective well(s). When following the directional vectors of the water movement in a (temporally invariant) velocity field, the distance traveled corresponds to a streamline. Once the three-dimensional velocity field of an aquifer is known, which assigns a velocity vector to any point within the stationary field, it is possible to determine one streamline for each of these points (which passes exactly through that point). Summarising all streamlines that lead to a depression (for example wells), one arrives at a spatial demarcation of the catchment area of the respective depression. If then all sources in that catchment area are captured, the water composition in the well (sink) can be derived.

Keywords Infiltration area Riverbank filtration · Residence time

\section{Hintergrund und Zielsetzung}

Die Reinigungsleistung im Untergrund (Uferfiltration) hängt stark von der Wasserqualität des Oberflächengewässers ab. Neben dem Einfluss der Oberflächenwasserqualität sind unter anderem die lokalen Gegebenheiten wie Milieubedingungen, Fließstrecke, Aufenthaltszeit und Qualität des vorhandenen Grundwassers wichtige Einflüsse. Für die Bearbeitungen zur Grundwasserqualität werden vielfach stationäre Systemzustände als Vereinfachung der tatsächlichen Strömungsverhältnisse betrachtet. Dabei ist zu beachten, dass die mithilfe eines numerischen Grundwassermodells berechneten stationären Systemzustände eine starke Vereinfachung der tatsächlichen Grundwassersituation darstellen, da diese in der Natur nur sehr selten vorkommen. Die doch einen quantitativen Vergleich unterschiedlicher Szenarien.

In diesem Artikel ist die Methodik zur Ausweisung von Brunneneinzugsgebieten und zur Abschätzung von Fließzeiten auf Basis eines 3D-stationären Grundwassermodells zusammengestellt. Zusätzlich ist die Methodik zur Erfassung von Quellen (Wasserherkunft) innerhalb eines Brunneneinzugsgebiets zur Abschätzung der Wasserzusammensetzung im Brunnen dargestellt.

\section{3D-stationäres Grundwassermodell}

Für die in diesem Artikel dargestellte Berechnung von Stromlinien bei Horizontalfilterbrunnen wurde ein bestehendes dreidimensionales, mit Oberflächengewässern gekoppeltes Modell der TU Wien herangezogen, welches mit der Modellierungssoftware SUTRA (Voss und Provost 2010) erstellt wurde. SUTRA steht für saturated-unsaturated transport und ist, wie schon aus dem Namen hervorgeht, in der Lage, die Wasserbewegung in der gesättigten und ungesättigten Bodenzone $\mathrm{zu}$ modellieren. Darüber hinaus bietet SUTRA die Möglichkeit, entweder Energietransport (Wärmebewegung) oder dispersiven Stofftransport zu beschreiben. Stromlinien können mit dieser Software nicht direkt berechnet werden. Die Transportgleichung wird von SUTRA durch einen hybriden Ansatz gelöst, zur Anwendung gelangt eine Mischform aus Finite-Elemente-Modellierung und integrierter Finite-Differenzen-Modellierung. Finite Elemente erlauben die Generierung von Netzgeometrien mit hoher, lokaler Genauigkeit während reine Finite-Differenzen-Modelle numerisch wesentlich effizienter $\mathrm{zu}$ lösen sind, jedoch nur beschränkt die Möglichkeit bieten, lokale Verfeinerungen des Netzes durchzuführen (Voss und Provost 2010, S. 7). Die räumliche Diskretisierung dreidimensionaler Mo- 


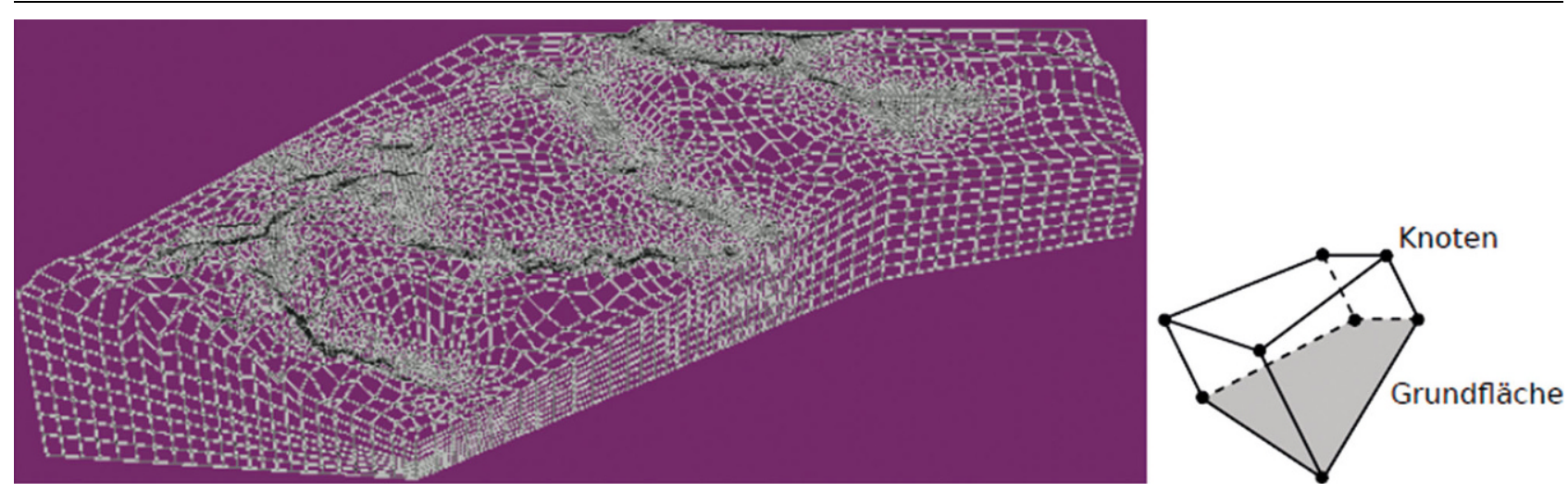

Abb. 1 Mögliche, unregelmäßige Netzgeometrie eines Grundwassermodells in SUTRA und allgemeine Form eines finiten Elements (Voss und Provost 2010)

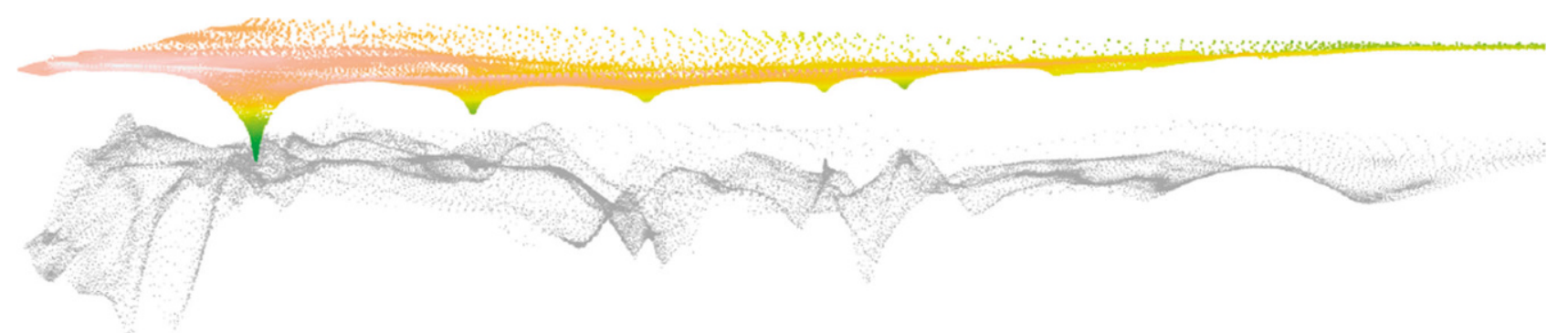

Abb. 2 Dreidimensionale Darstellung der interpolierten Grundwasseroberfläche (z-Richtung 100-fach überhöht). Die Höhe des Grundwasserstauers ist grau dargestellt

delle erfolgt in SUTRA durch hexagonale Elemente mit viereckiger Grundfläche (Abb. 1). In Summe besteht das vorliegende Modell aus ca. einer halben Million Knoten, verteilt auf 16 Tiefenstufen. Pro Tiefenstufe gibt es 34.951 Elemente. In x-Richtung beträgt die Ausdehnung des Modells knapp $12 \mathrm{~km}$, in y-Richtung etwa $9 \mathrm{~km}$. In Abb. 2 ist die dreidimensionale Darstellung der interpolierten Grundwasseroberfläche (z-Richtung 100-fach überhöht) dargestellt.

\section{Verfahren zur numerischen Integration von Stromlinien}

Folgt man in einem (zeitlich invarianten) Geschwindigkeitsfeld stets den Richtungsvektoren der Wasserbewegung, so entspricht der zurückgelegte Weg einer Stromlinie. Hat man nun Kenntnis über das dreidimensionale Geschwindigkeitsfeld eines Grundwasserleiters, das jedem beliebigen Punkt $\vec{p}=(p x, p y, p z)$ innerhalb des stationären Feldes einen Geschwindigkeitsvektor $\vec{v}=(v x, v y, v z)$ zuordnet, kann für jeden dieser Punkte $\vec{p}$ genau eine einzige Stromlinie bestimmt werden, die durch $\vec{p}$ geht. Mathematisch be- trachtet muss hierfür eine Differenzialgleichung erster Ordnung integriert werden, deren Lösung lediglich vom Anfangspunkt $\vec{p}$ der Stromlinie abhängt. Wird Wasser aus einem Grundwasserleiter gefördert, welcher seitlich durch Fließgewässer begrenzt ist und sonst keine weiteren Quellen und Senken aufweist, so werden die meisten Stromlinien am Gewässerrand beginnen und im Brunnen enden. Ziel ist es, diese Stromlinien aus dem im bestehenden dreidimensionalen Grundwassermodell gegebenen Geschwindigkeitsfeld und einem gewählten Punkt zu berechnen, um den Fließweg zu einem Brunnen zu erhalten. Meist liegt das Vektorfeld der Grundwasserfließgeschwindigkeiten nicht als stetige Funktion vor, sondern ist nur an bestimmten, diskreten Punkten auswertbar, beispielsweise an den Elementen eines Finite-Elemente-Modells. Das hat zur Folge, dass auch das Integral der Stromlinie nicht geschlossen lösbar ist und man auf Näherungsverfahren der numerischen Integration zurückgreifen muss (Papula 2011).

Das einfachste Verfahren hierfür ist das Eulersche Polygonzugverfahren, das verglichen mit z.B. dem RungeKutta-Verfahren ein deutlich schlech- teres Konsistenzverhalten zeigt. Zur Veranschaulichung soll die einfache Differenzialgleichung erster Ordnung

$$
y^{\prime}=\sin (x)^{2} \cdot y
$$

mit der analytischen Lösung

$$
y=2 \cdot \exp \left(\frac{x-\sin (x) \cos (x)}{2}\right)
$$

in dem Intervall $x \in[0,5]$ mit der festen Schrittweite $h=0,5$ mit beiden Methoden gelöst werden. Als Anfangswert wird $x_{0}=0$ gewählt. In weiterer Folge wird auf beide Verfahren der numerischen Integration von Anfangswertproblemen genauer eingegangen.

\subsection{Integration nach Euler}

Es sei das Anfangswertproblem

$$
y^{\prime}=f(x, y)
$$

gegeben, welches mit der Schrittweite $h>0$ und dem Startwert $y\left(x_{0}\right)=y_{0}$ gelöst werden soll. Somit ergibt sich für die ersten beiden Schritte

$$
y\left(x_{0}\right)=y_{0}
$$




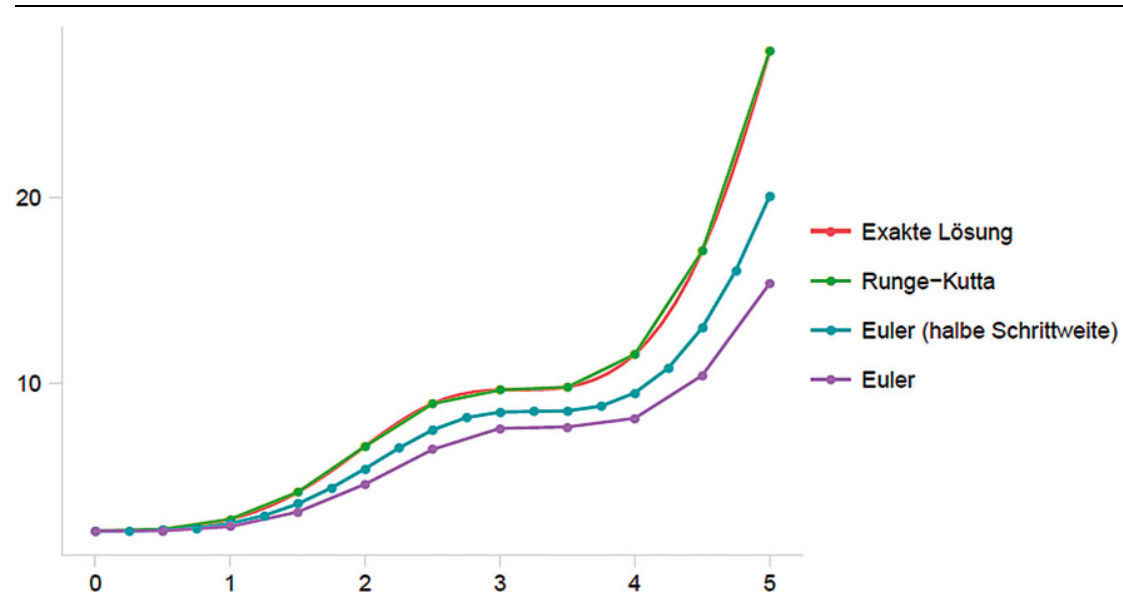

Abb. 3 Vergleich numerischer Integrationsverfahren für Anfangswertprobleme. Man beachte die großen lokalen Fehler des Euler-Verfahrens selbst bei Halbierung der Schrittweite
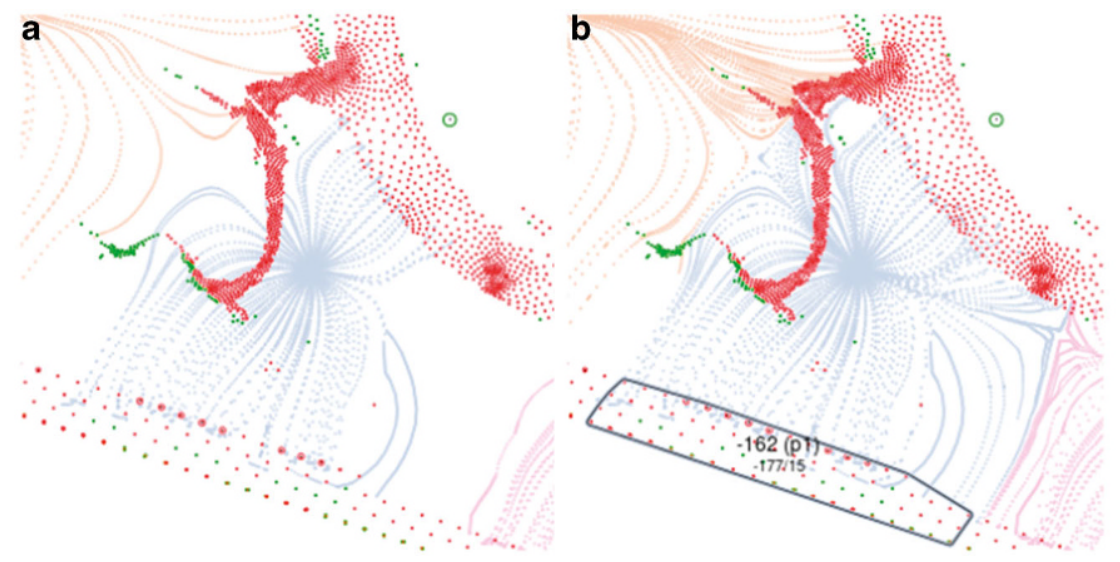

Abb. 4 Vorgangsweise bei der Abgrenzung von Einzugsgebieten

$$
y\left(x_{0}\right) \approx y_{1}=y_{k}+h \cdot f\left(x_{k}, y_{k}\right)
$$

und in allgemeiner Form

$$
\begin{gathered}
y\left(x_{k+1}\right) \approx y_{k+1}=y_{k}+h \cdot f\left(x_{k}, y_{k}\right) \\
(k=1,2, \ldots, n)
\end{gathered}
$$

Ausgehend von einem gewählten Anfangspunkt $P_{0}=\left(x_{0}, y_{0}\right)$ geht man $h$ Einheiten in Richtung der Tangentensteigung $f\left(x_{0}, y_{0}\right)$ und erhält einen Ordinatenwert $y_{1}$, welcher als Anfangswert für den darauffolgenden Schritt gewählt wird. Dieses Verfahren liefert nur eine grobe Näherung der exakten Lösungskurve, da zur Schätzung nur die Tangentensteigung im Ausgangspunkt berücksichtigt wird. Ausreichende Genauigkeiten sind nur mit hohem Rechenaufwand durch kleine Schrittweiten $h$ zu erhalten (Papula 2011, S. 475).

\subsection{Vergleich der Integrationsverfahren}

Wie in Abb. 3 zu sehen, weist das Euler-Verfahren bei gleicher Schrittweite deutlich größere lokale Fehler auf als das Runge-Kutta-Verfahren. Eine dem Runge-Kutta-Verfahren 4. Ordnung vergleichbare Genauigkeit erhält man für das explizite Euler-Verfahren erst bei ca. einem Tausendstel der Schrittweite $\left(h=5 \times 10^{-4}\right)$. Dies macht sich vor allem in Bereichen plötzlicher Richtungsänderung (z.B. große Grundwasserspiegelabsenkung in Brunnennähe bzw. Infiltration aus einem Fließgewässer) durch höhere, lokale Diskretisierungsfehler nachteilig bemerkbar. Deshalb wird hier zur Berechnung von Stromlinien nur das Runge-Kutta-Verfahren angewendet.

\section{Abgrenzung von Brunneneinzugsgebieten}

Fasst man alle Stromlinien, die zu einer Senke (z. B. Brunnen) gehen, zusammen, so gelangt man zu einer räumlichen Abgrenzung des Einzugsgebiets dieser Senke. Im zweidimensionalen Fall der Wasserbewegung $\mathrm{zu}$ einem Brunnen bezeichnet man jene Stromlinie, welche gerade noch im Brunnen endet, als Randstromlinie. Um diese aufzufinden, werden zunächst Stromlinien berechnet, welche in den Knoten des Horizontalfilterbrunnens beginnen. Dieser Vorgang ist in Abb. 4a für einen Brunnen gezeigt. Anschließend folgt eine manuelle Verdichtung in den Randbereichen des Brunneneinzugsgebietes analog zu Abb. 4b. Knoten, an denen Wasser ins Grundwasser infiltriert, sind rot dargestellt, Knoten, an denen Wasser austritt. grün. die zu integrierende Funktion in $s$ Zwischenstufen der Schrittweite $h$ aus. Es wird eine mittlere Steigung durch Gewichtung der $s$ Zwischenstufen gebildet. Das klassische Runge-Kutta-Verfahren besitzt die Ordnung $s=4$ und ist definiert mit

$$
y_{k+1}=y_{k}+1 / 6 \cdot\left(k_{1}+2 k_{2}+2 k_{3}+k_{4}\right)
$$

mit den vier Zwischenstufen

$$
\begin{aligned}
& k_{1}=h \cdot f\left(x_{k}, y_{k}\right) \\
& k_{2}=h \cdot f\left(x_{k}+h / 2, y_{k}+k_{1} / 2\right) \\
& k_{3}=h \cdot f\left(x_{k}+h / 2, y_{k}+k_{2} / 2\right) \\
& k_{4}=h \cdot f\left(x_{k}+h, y_{k}+k_{3}\right)
\end{aligned}
$$

Für einen Schritt sind somit vier Auswertungen der zu integrierenden Funktion notwendig (Papula 2011, S. 478).

\section{Ermittlung der Wasserzu- sammensetzung in den Brunnen} zugsgebiets, kann daraus die Wasserzusammensetzung im Brunnen (Senke) abgeleitet werden. In Abb. $4 \mathrm{~b}$ sind beispielsweise die Elemente von einem Modellrand zu einem Polygon $p 1$ zusammengefasst. Von dieser Seite strömen 1771/s in das Polygon und $15 \mathrm{l} / \mathrm{s}$ heraus, was einem Nettofluss von 162l/s in Richtung Brunnen entspricht. Fehler dieser Methode können darin liegen, dass ein Knoten immer nur einem Polygon/Brunnen zugeordnet werden kann. Aufgrund der räumlichen Diskretisierung des Modells und der
Erfasst man alle Quellen eines Ein- 
mitunter geringen Anzahl an Stromlinien kann es vorkommen, dass nicht alle Quellen eines Brunnens aufgefunden werden. $\mathrm{Zu}$ einer Abschätzung der Größe dieses Fehlers gelangt man, indem die dem Brunnen zuströmende Wassermenge der tatsächlich aus dem Brunnen entnommenen Wassermenge gegenübergestellt wird. Die daraus resultierende Differenz kann entweder auf eine fehlerhafte Zuordnung der Knoten zu Brunnen oder auf eine unzureichende Erfassung des Einzugsgebiets zurückgeführt werden.

\section{Abschätzung mittlerer Fließzeiten}

Mit einem dreidimensionalen, hydraulischen Modell kann für einen Punkt in der $x-y$-Koordinatenebene kein einzelner Wert der „Fließzeit“ angegeben werden, da über die ganze Höhe der gesättigten Bodenzone unendlich viele Stromlinien eine Messstelle durchströmen. Vielmehr besitzt die Variable Fließzeit $t$ eine Verteilung, welche durch Lageparameter (minimale Fließzeit $t_{\min }$, Medianwert der Fließzeit $t_{\mathrm{med}}$ ) und Streuungsparameter (Standardabweichung $\sigma$ ) beschrieben werden kann. Um über eventuelle, kleinräumig unbedeutende Variationen des Fließverhaltens in der Nähe einer Messstelle zu mitteln, werden Fließzeiten nicht nur für die exakten x-y-Koordinaten der Messstelle berechnet, sondern auch für fünf entlang eines Kreises mit dem Radius $r=20 \mathrm{~m}$ gelegene Punkte. Die Diskretisierung der Stromlinien in z-Richtung erfolgt analog zur Lage der gesättigten Modellknoten. Somit werden pro Messstelle im Mittel 40 Stromlinien berechnet. Die Relevanz eines Messwerts für die Wasserqualität im Horizontalfilterbrunnen ist umso höher, je näher die Messstelle beim Brunnen gelegen ist. Deshalb wird für jede Messstelle zusätzlich die Aufenthaltszeit des Wassers bis zum Brunnen angegeben. $\mathrm{Zu}$ einer gewichteten, mittleren Fließzeit $t_{\text {med }}^{\prime}$ pro Brunnen gelangt man, indem die einem finiten Element $i$ zuordenbare Fließzeit $t_{\text {med }}$, mit der Quellenstärke $Q_{i}$ gewichtet wird. Da eine eindimensionale Stromlinie mit dem Durchmesser 0 definitionsgemäß keinen Durchfluss haben kann, werden die Volumenströme für Bündel an Stromlinien ähnlicher Fließzeit und Elemente mit vergleichbarer Quellenstärke ermittelt.

$$
\bar{t}_{\text {med }}=\frac{\sum_{i=1}^{n} Q_{i} \cdot t_{\text {med }, i}}{\sum_{i=1}^{n} Q_{i}}
$$

$\bar{t}_{\text {med }} / s \quad$ gewichtete, mittlere Fließzeit (T)

n/- Anzahl der Gewichte (Quellenpolygone) (1)

$Q_{i} / 1 \mathrm{~s}^{-1} \quad$ Zufluss zum Brunnen aus der $i$-ten Quelle $\left(\mathrm{L}^{3} \mathrm{~T}^{-1}\right)$

$t_{\text {med }, i} / s \quad$ Medianwert der Fließzeit von der $i$-ten Quelle zum Brunnen (T)

Für Stromlinien, welche am Modellrand „enden“, ist keinesfalls anzunehmen, dass die Fließzeit mit der Aufenthaltszeit des Uferfiltrats gleichzusetzen ist. Diese ist wesentlich höher anzunehmen, denn Fließgewässer entsprechender Größe weisen einen ausgeprägten Grundwasserbegleitstrom auf. Aufgrund des Modellrands werden die Stromlinien in diesem Bereich abgeschnitten. Die absoluten Beträge der angegebenen Fließzeiten stimmen deshalb nicht mit den tatsächlichen Fließzeiten überein. Betrachtet man jedoch die Änderung der Fließzeiten zwischen unterschiedlichen Szenarien, so wird dieser Nullpunktfehler, welcher durch die Unkenntnis des wahren Beginns der Stromlinie auftritt, durch Differenzenbildung eliminiert bzw. zumindest reduziert.

\section{Schlussfolgerung}

Mithilfe der Berechnung von Stromlinien können gerade bei Uferfiltratbrunnen der Einzugsbereich und die Wasserherkunft ermittelt werden. Insbesonde- re in den Randbereichen der Einzugsgebiete bilden sich Wasserscheiden mit sehr flachen Gradienten und geringen Wasserbewegungen aus. In der Realität sind die Einzugsgebiete nicht durch eine exakt definierbare Grenze getrennt, vielmehr bilden sich dort Zonen mit geringen Fließgeschwindigkeiten aus, die je nach hydrologischem Zustand im Oberflächengewässer variieren können. Grundwassermessstellen zur Wasserqualitätsüberwachung in diesen Randbereichen besitzen nur eine geringe Aussagekraft in Hinblick auf die Wasserqualität im Brunnen. Werden die Einzugsgebiete mit den berechneten Stromlinien mit ausreichender Präzision erfasst, so entspricht die ermittelte Wasserzusammensetzung der zuströmenden Wassermenge eines Brunnens der tatsächlich gemessenen Entnahmemenge im Brunnen mit nur geringen Abweichungen. Die Anteile der einzelnen Quellen (Wasserherkunft) eines Brunnens können somit deutlich verbessert interpretiert werden. Durch die Ermittlung der Fließzeiten der einzelnen Mengenanteile einer Quelle bis zum Brunnen kann zusätzlich eine vertiefende Information über die minimale und mittlere Fließzeit dargestellt werden.

Funding Open access funding provided by University of Natural Resources and Life Sciences Vienna (BOKU).

Open Access Dieser Artikel wird unter der Creative Commons Namensnennung 4.0 International Lizenz (http:// creativecommons.org/licenses/by/4. $0 /$ deed.de) veröffentlicht, welche die Nutzung, Vervielfältigung, Bearbeitung, Verbreitung und Wiedergabe in jeglichem Medium und Format erlaubt, sofern Sie den/die ursprünglichen $\mathrm{Au}$ tor(en) und die Quelle ordnungsgemäß nennen, einen Link zur Creative Commons Lizenz beifügen und angeben, ob Änderungen vorgenommen wurden. 


\section{Originalarbeit}

\section{Literatur}

Papula, L. (2011): Mathematik für Ingenieure und Naturwissenschaftler Band 2: Ein Lehrund Arbeitsbuch für das Grundstudium. View-

eg+Teubner Verlag/Springer Fachmedien, Wiesbaden, Germany

Voss, C. I., Provost, A. M. (2010): SUTRA-A

Model for Saturated-Unsaturated, VariableDensity Ground-Water Flow wirth Solute of

Energy Transport. U.S. Department of the In-

terior, U.S. Geological Survey, Reston, Virginia;

USGS Numbered Series 2002-4231; https://doi.

org/10.3133/wri024231

Hinweis des Verlags Der Verlag bleibt in Hinblick auf geografische Zuordnungen und Gebietsbezeichnungen in veröffentlichten Karten und Institutsadressen neutral. 